\section{IN BRIEF}

\section{IMMUNITY}

\section{Distinct macrophage subsets in the human heart}

The human heart contains C-C chemokine receptor type 2 $(\mathrm{CCR} 2)^{+}$and $\mathrm{CCR}^{-}$macrophage subsets with distinct origins and functions, similar to the macrophage heterogeneity found in mouse hearts. Investigators used transmural left ventricular (LV) myocardial specimens collected during heart transplantation or the implantation of an LV assist device in patients with dilated or ischaemic cardiomyopathy. Analysis of sex-mismatched heart transplantation recipients showed that $\mathrm{CCR2}^{+}$macrophages are maintained through monocyte recruitment and proliferation. By contrast, $\mathrm{CCR}^{-}$macrophages are replenished exclusively by local proliferation and are a tissue-resident population. The subsets of macrophages have distinct functions, analogous to the inflammatory $\mathrm{CCR}^{+}$and reparative CCR2 ${ }^{-}$macrophages in mouse hearts. Of note, $\mathrm{CCR}^{+}$macrophage abundance is associated with systolic function and LV remodelling in patients with heart failure. ORIGINAL ARTICLE Bajpai, G. et al. The human heart contains distinct macrophage subsets with divergent origins and functions. Nat. Med. https://doi.org/10.1038/ s41591-018-0059-x (2018)

\section{LIPIDS}

\section{LIMA1 variant influences cholesterol absorption}

A rare frameshift mutation (K306fs) in LIMA1 (also known as EPLIN or SREBP3), which encodes the LIM domain and actin-binding protein 1 (LIMA1) causes reduced cholesterol absorption in the gut and low levels of serum LDL cholesterol. The mutation was identified in a Chinese family of Kazakh ethnicity. In mice, LIMA1 was found to be expressed mainly in the brush border membrane of the small intestine. LIMA1 binds to NPC1-like intracellular cholesterol transporter 1 (NPC1L1) and facilitates cholesterol uptake. Mice with intestine-specific LIMA1 deficiency had reduced cholesterol absorption and were resistant to diet-induced hypercholesterolaemia, recapitulating the phenotype of the LIMA1-K306fs variant in humans. The researchers suggest that inhibiting LIMA1 might provide a therapeutic approach to lowering LDL-cholesterol levels that is complementary to the use of statins, ezetimibe (which inhibits NPC1L1), or PCSK9 inhibitors.

ORIGINAL ARTICLE Zhang, Y.-Y. et al. A LIMA1 variant promotes low plasma LDL cholesterol and decreases intestinal cholesterol absorption. Science 360, 1087-1092 (2018)

\section{RISK FACTORS}

\section{Erectile dysfunction predicts CVD events}

Vascular erectile dysfunction (ED) is a significant predictor of cardiovascular disease (CVD) events, according to data from MESA. Investigators identified 1,757 individuals with ED on the basis of an answer to a questionnaire and who had no history of CVD events (myocardial infarction, stroke, resuscitated cardiac arrest, and stroke or cardiac death). During 3.8 years of follow-up, the rate of CVD events was higher in participants with ED than in those without $\mathrm{ED}(6.3 \%$ versus $2.6 \% ; P<0.001)$. In a model fully adjusted for traditional CVD risk factors, depression, and $\beta$-blocker use, ED was a significant predictor of CVD events (HR 1.9, 95\% Cl 1.1-3.4). ED and CVD have shared risk factors (obesity, hypertension, metabolic syndrome, diabetes mellitus, and smoking) and pathological mechanisms (endothelial dysfunction, inflammation, and atherosclerosis). The presence of ED could, therefore, have important implications for CVD risk stratification in middle-aged men.

ORIGINAL ARTICLE Uddin, S. M. I. et al. Erectile dysfunction as an independent predictor of future cardiovascular events: the Multi-Ethnic Study of Atherosclerosis. Circulation https://doi.org/10.1161/CIRCULATIONAHA.118.033990 (2018)

\title{
Dual-therapy stent shows promise
}

A stent that combines a

bioabsorbable polymer, sirolimus elution to prevent restenosis, and murine anti-CD34 antibodies to capture circulating endothelial progenitor cells (EPCs) with the aim of accelerating healing at the site of percutaneous coronary intervention (PCI) has shown noninferiority to everolimus-eluting stents (EES) for 1-year target-vessel failure, according to findings from the randomized, pivotal, registration trial, Japan-USA HARMONEE.

Despite improvements in the safety and efficacy of drug-eluting stents, safety concerns remain, in particular with respect to stent thrombosis. Delayed endothelialization after DES implantation might be a substrate of stent thrombosis, which prompted the development of stents coated with anti-CD34 antibodies to capture bone marrow-derived CD $34^{+}$ EPCs. The hypothesis was that captured EPCs would differentiate into endothelial cells, thereby facilitating endothelial coverage of the stent and promoting early healing after stent implantation.

The primary clinical end point of the trial was noninferior 1-year target-vessel failure with the Combo stent compared with EES in patients undergoing PCI for ischaemic coronary disease and non-ST-segment elevation acute coronary syndromes. Superior strut coverage assessed by optical coherence tomography (OCT) was used as a surrogate marker of the EPC-capture technology (primary mechanistic end point).

At 1 year after PCI, the Combo stent met the noninferiority criteria for target-vessel failure, with a rate of $7.0 \%$ (20 of 287 patients) compared with $4.2 \%$ (12 of 285 patients) for EES $(P=0.02)$. No cardiac deaths occurred, one stent thrombosis was observed in the EES group, and angiographic late lumen loss was low and similar with both stent types. The mechanistic OCT analysis showed that strut coverage

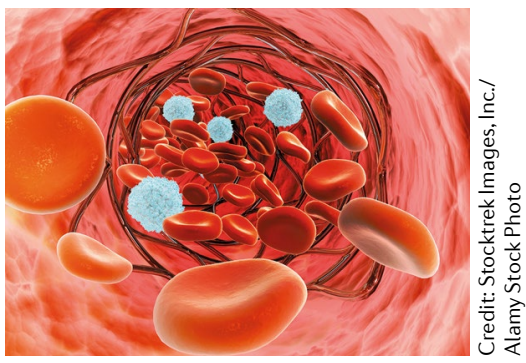

with healthy tissue $(>40 \mu \mathrm{m})$ was significantly greater with the Combo stent $(91.3 \%)$ than with EES (74.8\%; $P<0.001)$, with homogeneous tissue in $81.2 \%$ versus $68.8 \%$ of the analysed lesions, respectively. Of note, almost all struts were covered with either the Combo stent or EES (99.2\% versus 98.8\%; $P=0.02$ ), and neointimal hyperplasia thickness was significantly greater with the Combo stent than with EES $(0.180 \mathrm{~mm}$ versus $0.107 \mathrm{~mm}$; $P<0.001)$.

In summary, the Combo stent was noninferior to EES for 1-year target-vessel failure and late lumen loss, with superior strut coverage with more homogeneous tissue. Nevertheless, the trial investigators caution that the patient cohort had a less complex clinical history and anatomy than expected, which led to the observed 1-year target-vessel failure rate of $4.2 \%$ with EES being much lower than the protocol-assumed rate of $9.0 \%$. Therefore, further studies are needed to confirm the noninferiority of the Combo stent to EES in patients with a more complex medical history and anatomy.

Irene Fernández-Ruiz

ORIGINAL ARTICLE Saito, S. et al. Japan-United States of America Harmonized Assessment by Randomized Multicentre Study of OrbusNEich's Combo StEnt (Japan-USA HARMONEE) study: primary results of the pivotal registration study of combined endothelial progenitor cell capture and drug-eluting stent in patients with ischaemic coronary disease and non-ST-elevation acute coronary syndrome. Eur. Heart J. https://doi.org/ 10.1093/eurheartj/ehy275 (2018) FURTHER READING Ong, D. S. et al. Causes, assessment, and treatment of stent thrombosis -intravascular imaging insights. Nat. Rev. Cardiol. 12, 325-336 (2015) 